\title{
TEXTKRITISCHES ZU DEN EPIGRAMMEN MELEAGERS (II)*
}

\author{
THOMAS GÄRTNER \\ Universidad de Colonia \\ th-gaertner@gmx.de
}

\section{TEXTUAL CRITICISM ON THE EPIGRAMS OF MELEAGER (II) CRÍTICA TEXTUAL DE LOS EPIGRAMAS DE MELEAGRO (II)}

In dem Aufsatz wird eine Vielzahl textkritischer Einzelprobleme aus den Epigrammen Meleagers besprochen in beständiger Auseinandersetzung mit der kommentierten Ausgabe von Gow und Page.

Schlüsselwörter: Meleager; Epigrammatik; Textkritik; Anthologia Palatina.
In the present paper a number of questions of textual criticism concerning the epigrams of Meleager is raised. The commented edition of Gow and Page is constantly used as a starting point of the discussion.

Keywords: Meleager; epigrams; textual criticism; Anthologia Palatina.

En este artículo se plantean una serie de cuestiones de crítica textual, concernientes a los epigramas de Meleagro. La edición comentada de Gow y Page es tomada como punto de partida de la discusión.

Palabras clave: Meleagro; epigramas; crítica textual; Antología Palatina.

$$
A P \mathrm{~V} 184=H E 4370-4377
$$

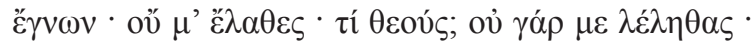

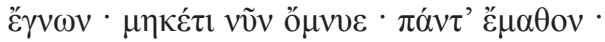

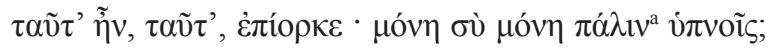

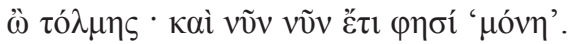

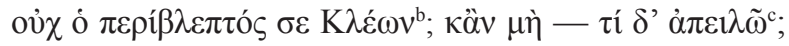

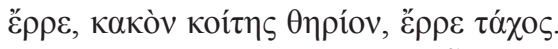

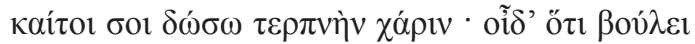

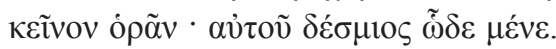

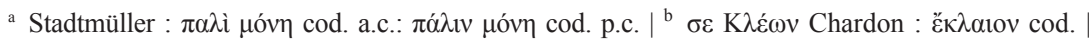

c Seidler : à $\pi \varepsilon i ́ \delta \omega$ cod.

\footnotetext{
* Este artículo es la continuación del publicado en Emerita LXXV,1, 2007, pp. 93-112.
} 
Gow-Page akzeptieren Stadtmüllers Umstellung aus metrischen Gründen, um die Hiatkürzung nach dem fünften $\mathrm{Fu} ß \mathrm{zu}$ vermeiden, die nach den Ausführungen zu $H E 4350$ gleichwohl eine kaum zu bestreitende Parallele in $A P$

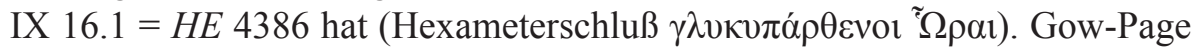
nehmen offenbar an, daß der Autor eine solche, seinem Sprachgebrauch an sich entsprechende Hiatkürzung dann vermeiden muß, wenn sie sich durch eine leichte Umstellung umgehen läßt.

Hinzuzunehmen ist nun freilich die Tatsache, daß $\pi \dot{\alpha} \lambda \imath v$ seinerseits inhaltliche Schwierigkeiten aufwirft, wie Gow-Page zugeben («the point is by no means clear»). Das offenkundig als Adverb zu virvoĩ gebrauchte Wort auf eine Wiederholung des Adjektivs $\mu$ óv $\eta$ zu beziehen (Waltz), setzt eine allzu kühne Brachylogie voraus. Gow-Pages Alternativdeutung «you claim to be sleeping alone again (as you did in the past, before these recent amours)» überzeugt nicht, insofern die Äußerung $\mu$ óv $\eta \pi \alpha ́ \lambda ı v ~ v i \pi v \tilde{~ d a n n ~ g e r a d e ~ w e g e n ~}$

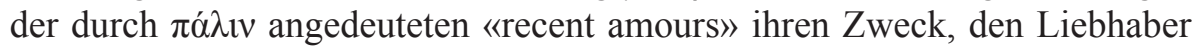
$\mathrm{zu}$ beruhigen, von vorneherein verfehlen müßte.

Eher dürfte die Dame versichert haben, schon lange alleine zu schlafen, d.h. schon lange Zeit keinen Liebhaber mehr zu haben. Man wird, von der nicht korrigierten Lesart ausgehend, schreiben:

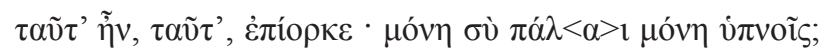

In Anbetracht der vom Sprecher gemachten Entdeckung erhält dieses

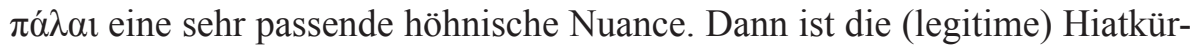
zung auch nicht mehr durch einfache Umstellung zu beseitigen.

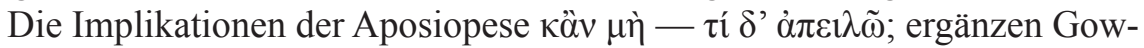
Page mit «Is not Cleon your lover? And even if he is not ...». Aber ein solches Zugeständnis paßt überhaupt nicht zur zornigen Stimmung des Sprechers,

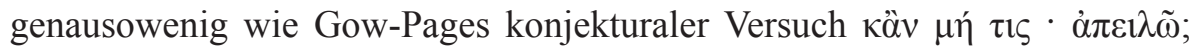
«and even if nobody ... - I threaten you, begone».

Insofern die offenbar als dem Sprecher gegenüberstehend zu denkende

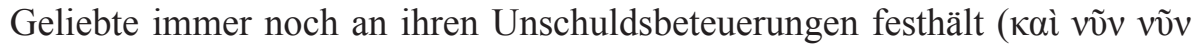

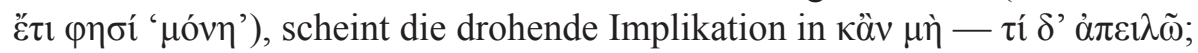
vielmehr etwa folgende zu sein: «Und wenn du es nicht (zugibst, werde ich es aus dir herausprügeln)», vgl. $A L 446 \mathrm{R} .=444 \mathrm{SB}=$ Sen. (?), Epigr. 54.3: Nempe parum casta es, nempe es deprensa: negabis? Zunächst denkt der Sprecher an ein solches "peinliches Verhör», dann bricht er seine Drohungen 


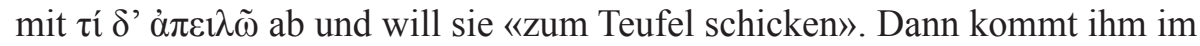
Schlußdistichon der Gedanke, daß sie dann zu seinem Rivalen gehen wird, und entschließt sich, sie zu «inhaftieren».

$$
A P \text { XII } 114=H E 4390 \mathrm{~s} .
$$

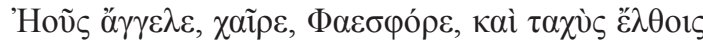

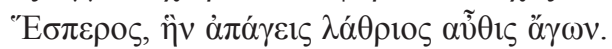

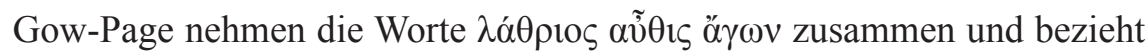
sie auf ein «clandestine rendezvous». Aber die näheren Umstände der Rückkunft der Geliebten dürften den Sprecher hier kaum interessieren, und auch

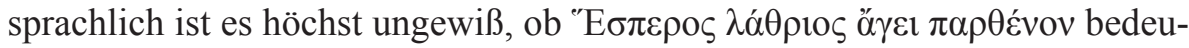
ten kann «der Hesperos führt eine junge Frau herbei, ohne daß diese bemerkt wird» (der Aufgang des Abendsterns kann ja als solcher unmöglich «heimlich» geschehen). Gegen dieses sprachliche Bedenken hilft auch nicht SHell.

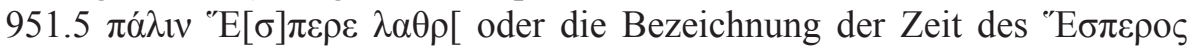
als $\lambda \alpha \dot{\theta} \theta \rho ı \varsigma \varsigma \emptyset \eta \eta$ bei Musae. 109 (vgl. Kost z.St.); eine zu der letztgenannten Stelle parallele Verwendung ("E $\sigma \pi \varepsilon \rho \circ \varsigma \lambda \alpha \dot{\alpha} \theta \rho ı \varsigma=$ "der Abend, Zeit erotischer Heimlichkeiten') scheitert daran, daß $\lambda \alpha \dot{\theta} \theta \rho ı \varsigma$ in der in unserem Epigramm vorliegenden Wortstellung nicht attributiv gedeutet werden kann. Stilistisch erwartet man einen Begriff, der sich mit ả $\pi \alpha ́ \gamma \varepsilon 1 \zeta$ verbindet zu einer formal befriedigenden Antithese zu $\alpha \tilde{\theta} \theta ı \check{\alpha} \gamma \omega v$ :

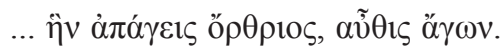

... die zurückführend, die du am frühen Morgen wegführst.

$$
A P \text { XII } 94=H E 4392-4397
$$

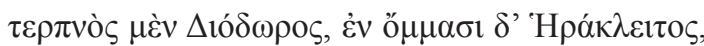

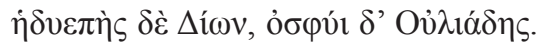

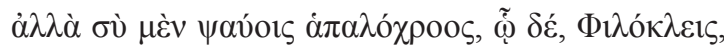

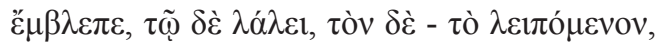
$\dot{\omega} \varsigma \gamma v \tilde{\varphi} \varsigma$, oĩo

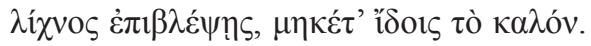

Gow-Page arbeiten richtig heraus, daß im ersten Glied der Aufzählung der

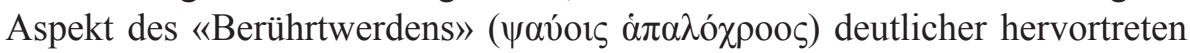


muß als durch unspezifisches $\tau \varepsilon \rho \pi v o ̀ \varsigma ~ \mu \varepsilon ́ v$. Fraglich erscheint aber, ob Grae-

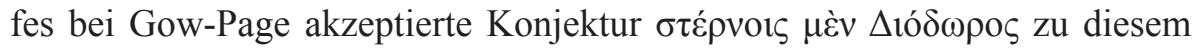
Zweck geeignet ist. Wenn sich $\sigma \tau \varepsilon \dot{p} v o r \zeta$ auf die Brust des Knaben beziehen sollte (vgl. die von Gow-Page zitierte Parallele AP XII 96.3 s. $=H E 3788 \mathrm{~s}$.

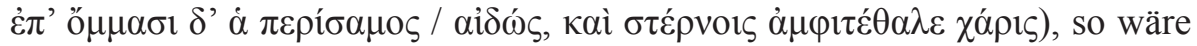
der Ausdruck höchst mißverständlich und unklar, weil sich $\sigma \tau \varepsilon \dot{\rho} \rho v o r \varsigma$ auf ein

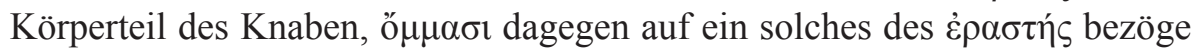

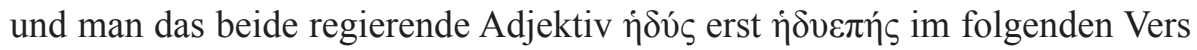

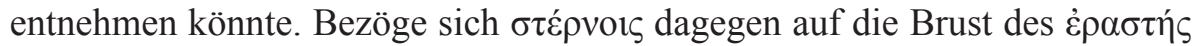
(was wohl wahrscheinlicher wäre), dann bezeichnen $\sigma \tau \varepsilon \dot{\varepsilon} p v o r \varsigma$ und ö $\mu \mu \alpha \sigma$ zwar Körperteile derselben Person, aber es liegt nahe, $\sigma \tau \varepsilon \dot{\rho} p v o ı \varsigma$ auszudeuten in dem unspezifischen Sinne «Diodoros ist dem Herzen lieb» (vgl. LSJ s.u.

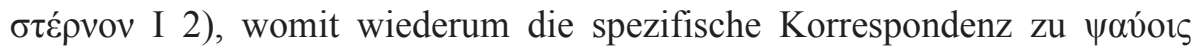
$\dot{\alpha} \pi \alpha \lambda \chi_{0} \rho 00 \varsigma$ verlorenginge.

Rätlicher erscheint es demnach, an dem überlieferten $\tau \varepsilon \rho \pi v o ́ \varsigma$ festzuhalten; dann sind die ersten beiden Glieder ohne Rekurs auf das dritte verständlich, und bei ỏ $\sigma \varphi v ́ r$ wird kaum ein Leser nachgrübeln, ob er noch einmal $\tau \varepsilon \rho \pi v o ́ s$

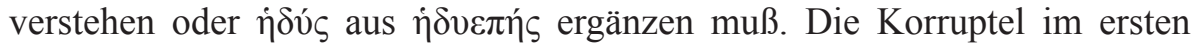
Glied könnte dann nur hinter der Partikel $\mu \varepsilon \dot{v}$ zu suchen sein. Diese Partikel ist nicht erforderlich. Denn in Vers 3 korrespondiert $\mu \varepsilon \dot{v} v$ wahrscheinlich mit

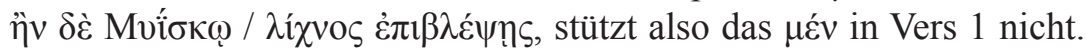

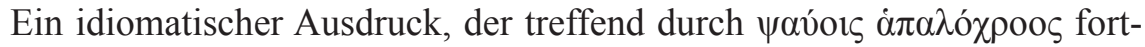

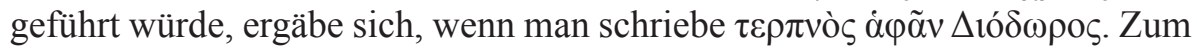
Gebrauch des aktiven Infinitivs vgl. Kuehner-Gerth II 15 f.; zur erotischen

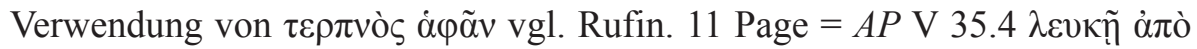

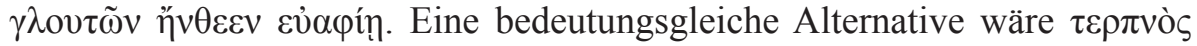

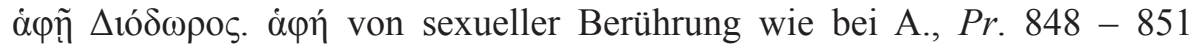

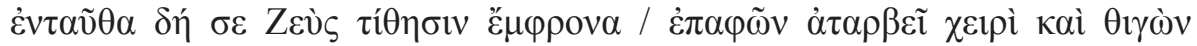

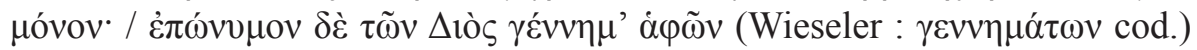

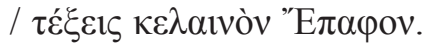

Eine weitere Schwierigkeit liegt in dem von $\gamma v \tilde{\omega} \varsigma$ abhängigen Frage-

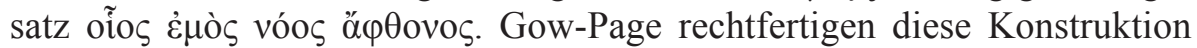
mit einem Verweis auf $L S J$ s.v. oĩos II 7. Dort finden sich die Belegstellen

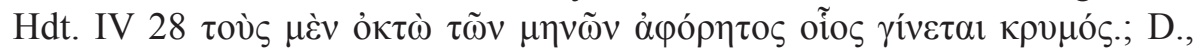

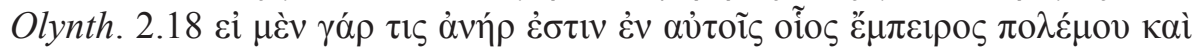

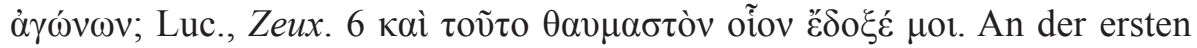
und dritten Stelle wirken wohl inverse Attraktionen vom Nebensatz auf den 
Hauptsatz, bei Demosthenes ist nichts anderes gemeint als talis uir qualis peritus. Aus keiner dieser Parallelen ergibt sich jedoch ein Beleg, daß ein

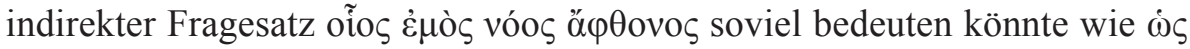
$\ddot{\alpha} \varphi \theta$ ovos દ̇uòs vóos.

Inhaltlich sähe man auch in diesem abhängigen Fragesatz gern einen Bezug auf den Angesprochenen, der nach dem Vorgen die verschiedenen Knaben genießen, aber nach dem Folgenden Myiskos nicht einmal lüstern anschauen

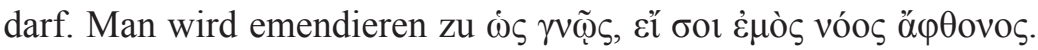

$$
A P \text { XII } 164=H E 4428-4431
$$

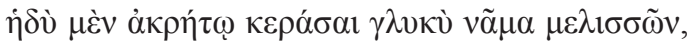

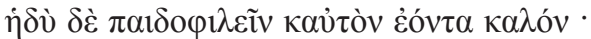

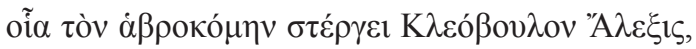

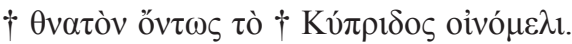

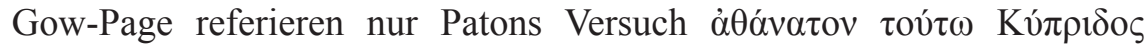
oivó $\mu \varepsilon \lambda$. Der begrifflich «sterblich» scheint aber gut zu passen als Antithe-

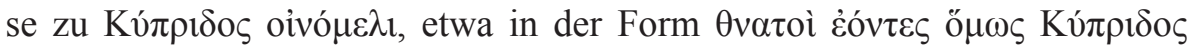
oivó $\mu \varepsilon \lambda \imath$ (sc. cióív).

$$
A P \text { XII } 52=H E 4432-4437
$$

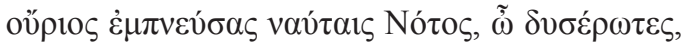

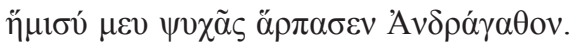

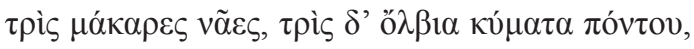

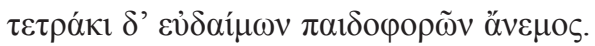

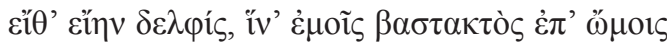

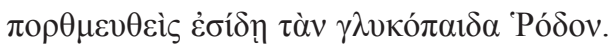

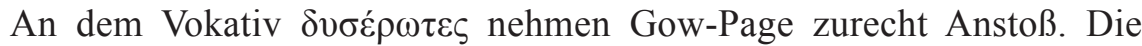

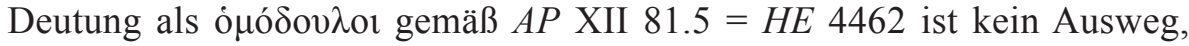

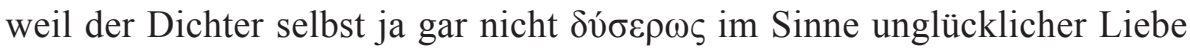
ist, sondern nur von seinem Geliebten durch dessen Aufbruch zu einer

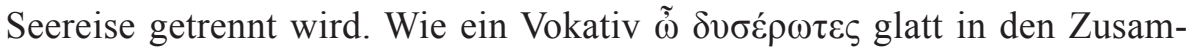
menhang eingefügt werden kann, zeigt exemplarisch auch das Adespoton $A P$ XII $79=$ HE 3694 - 3697: 


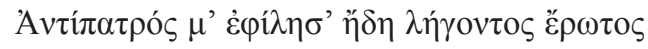

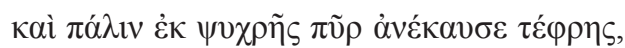

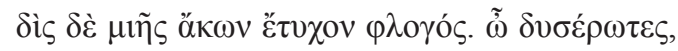

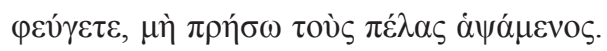

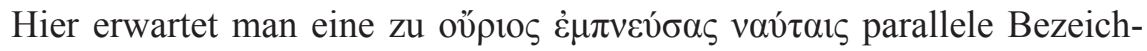
nung der Implikationen des Nótoৎ für die Liebenden:

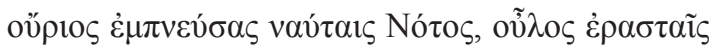

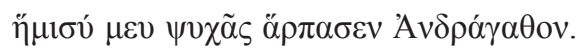

Der Gedichteingang wird imitiert bei Ou., Ep. 13.9-12:

Raptus es hinc praeceps, et qui tua uela uocaret,

Quem cuperent nautae, non ego, uentus erat.

Ventus erat nautis aptus, non aptus amanti.

Soluor ab amplexu, Protesilae, tuo.

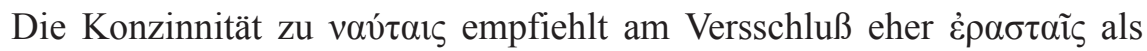

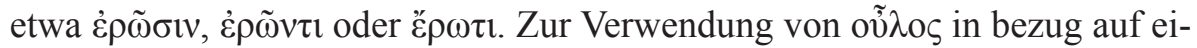
nen Wind vgl. den Hexameterschluß aus den Bassarika des Dionysius (fr. 22

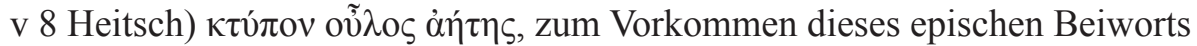

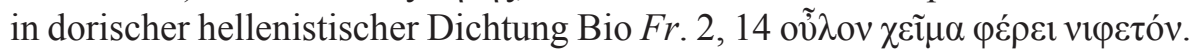

$$
A P \text { XII } 54=H E 4438-4441
$$

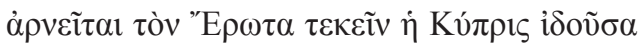

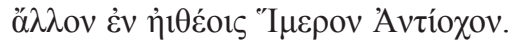

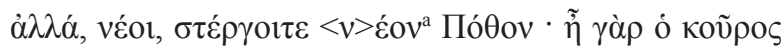

$\varepsilon$

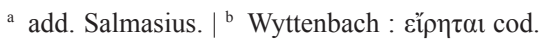

Der Wunsch, die jungen Leute mögen den «neuen Eros» lieben, wirkt relativ schwach, nachdem dessen Akzeptanz in diesem Kreis bereits durch den

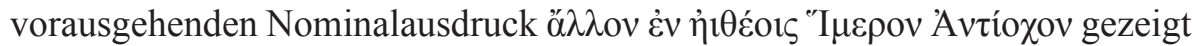
worden ist. Außerdem befremdet das unpointierte Polyptoton véor ... véov. 
Das Gedicht gewinnt an Geschlossenheit und enkomiastischem Ausdruckswert, wenn auch im zweiten Distichon über die Akzeptanz des Antiochos nicht unter den Menschen, sondern unter den Göttern gesprochen wird. So erhält das am Anfang beschriebene Gebaren der Venus eine passende Fortführung:

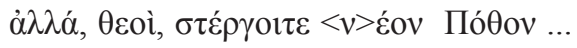

So möget ihr, Götter, euch mit dem neuen Eros abfinden...

$A P$ XII $158=H E 4496-4503$

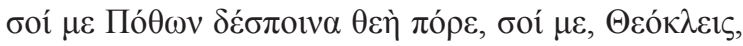

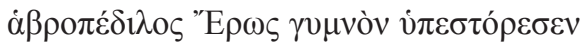

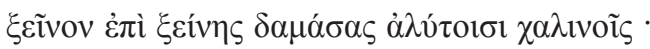

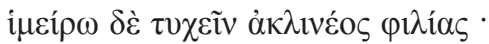

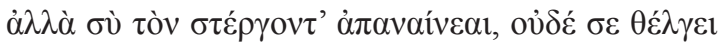

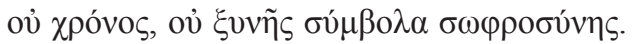

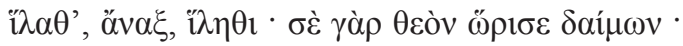

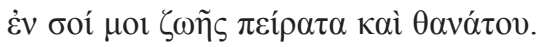

Gow-Page geben zu, daß der Begriff $\sigma \omega \varphi \rho \sigma^{\prime} v v_{\eta}$ «surprising» ist, erklären ihn aber doch, und zwar zunächst in dem Sinne «that they have long since come to their senses and have been enjoying a mutual friendship»; aber das damit verbundende nicht-erotische Verständnis von $\varphi \imath \lambda i ́ \alpha$ verbietet sich, weil der Sprecher seine Absichten mit $\gamma v \mu v o ̀ v ~ v i \pi \varepsilon \sigma \tau o ́ \rho \varepsilon \sigma \varepsilon v$ deutlich genug bekundet und gerade aus diesem seruitium amoris seine Absicht i $\mu \varepsilon i ́ \rho \omega ~ \delta \grave{\varepsilon}$

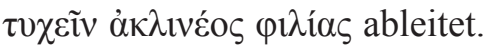

Alternativ verstehen Gow-Page $\sigma \omega \varphi \rho \sigma^{\prime} v \eta$ im Sinne eines «settled and

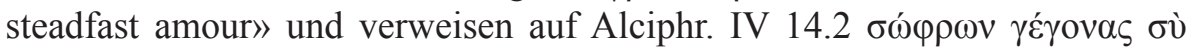

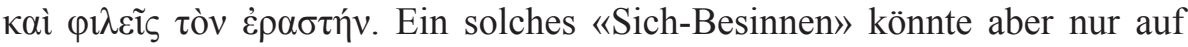
der Seite des widerspenstigen Knaben liegen und insofern kaum das Attribut

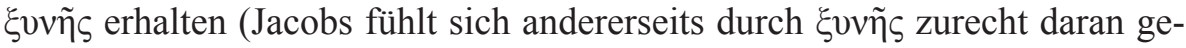
hindert, bei $\sigma \omega \varphi \rho о \sigma o ́ v \eta$ an die «amantis spectata uirtus», also an die Tugend des Sprechers, zu denken). Auch begreift man nicht, wie gesagt sein kann, daß die «Unterpfänder» $(\sigma 0 ́ \mu \beta o \lambda \alpha)$ einer so verstandenen $\sigma \omega \varphi \rho o \sigma u ́ v \eta$ den Widerspenstigen nicht besänftigten, wenn er offenbar über solche $\sigma \omega \varphi \rho \circ \sigma v ́ v \eta$ schlechterdings nicht verfügte. 


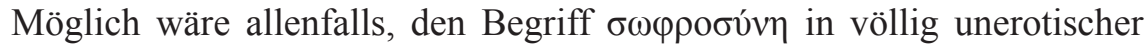
Weise auf eine politische Grundhaltung zu beziehen (die dann in ihrer oligarchischen Ausrichtung an die Theognideen erinnern würde), so daß diese gemeinsame Grundhaltung als ein Argument für ein erotisches Entgegenkommen herangezogen würde. In Anbetracht der herausgestellten Eindeutigkeit des erotischen Ansinnens und der konservativen Werthaftigkeit der $\sigma \omega \varphi \rho о \sigma v ́ v \eta$ wäre dies jedoch einigermaßen grotesk.

Passenderweise sollte hingegen die Rede sein von den «Unterpfändern

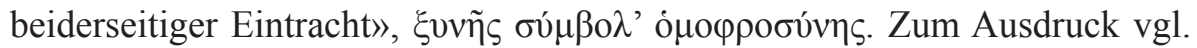

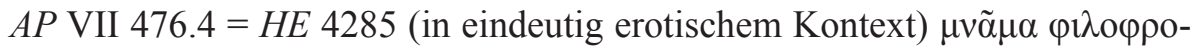

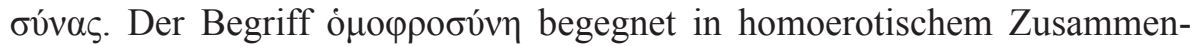

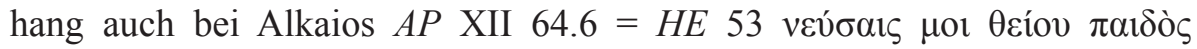
oн

$$
A P \text { XII } 141=H E 4510-4515
$$

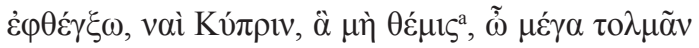

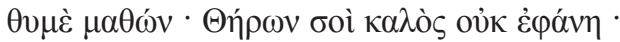

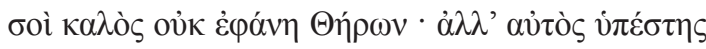

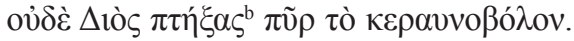

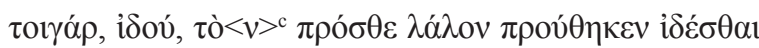

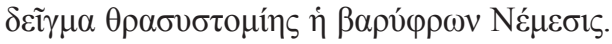

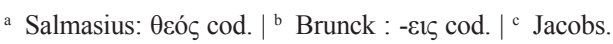

Gow-Page verteidigen das Kompositum vं $\pi \dot{\sigma} \sigma \tau \uparrow$ zurecht mit LSJ s.v.

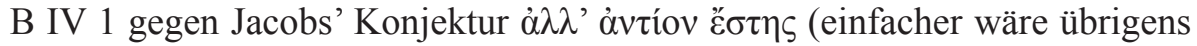

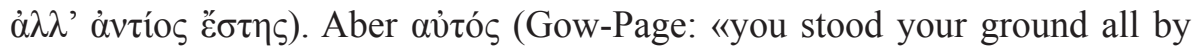
yourself») ist kaum zu halten, weil der Gedanke an fremde Unterstützung fernliegt. Man gewinnt einen pointierten Gegenbegriff zu dem folgenden, wörtlich $\mathrm{zu}$ verstehenden $\pi \tau \dot{\eta} \xi \alpha \varsigma$ («sich niederduckend vor»), wenn man

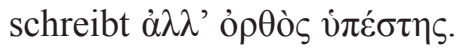

Im Schlußdistichon stört man sich an dem blassen partikelhaften Ge-

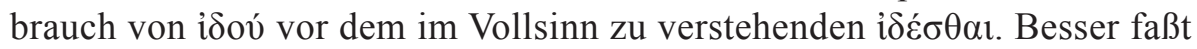
man schon den Imperativ als eigentliches Verb auf und verbindet $\tau$ or $\gamma \alpha \dot{\alpha} \rho$

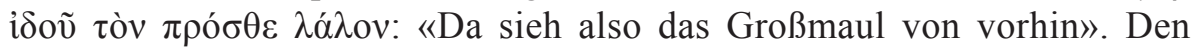
Rest des Distichons macht man zu einem befriedigenden explizierenden Schlußsatz, indem man das dort dann fehlende Objekt nachträglich einführt: 


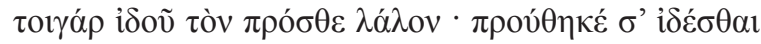

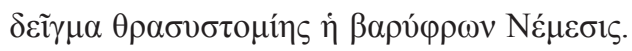

(oder allenfalls mit Elision in der Hauptdihaerese des Pentameters: $\pi \rho \circ \theta \theta\rceil \kappa \varepsilon v$

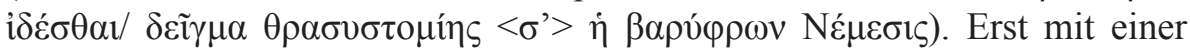
solchen abschließenden Apostrophe an den $\theta v \mu o ́ s$ erhält das mit $\dot{\varepsilon} \varphi \theta \dot{\varepsilon} \gamma \xi \omega$ beginnende Gedicht seine formale Abrundung.

$$
A P \text { XII } 165=H E 4520-4523
$$

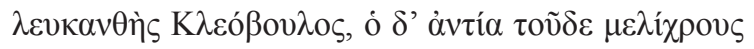

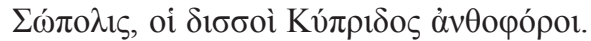

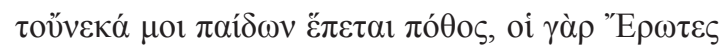

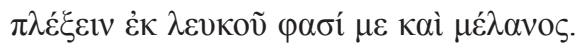

Die Eroten werden keine aitiologische Aussage machen über das «Geflecht», aus welchem das dichterische Ich besteht, die man zudem mit einem Infinitiv Aorist mühsam an den Zusammenhang adaptieren müßte ( $\pi \lambda \varepsilon \dot{\varepsilon} \xi \alpha$

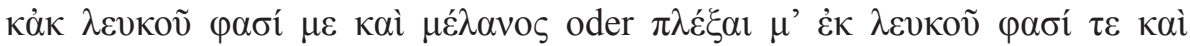

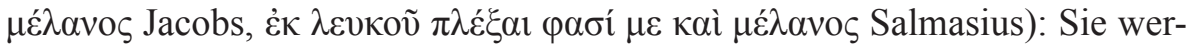
den einfach gesagt haben, mit welchem «Feuer» sie Meleager in Brand zu setzen gedenken:

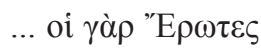

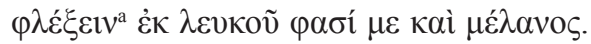

a Rossbach.

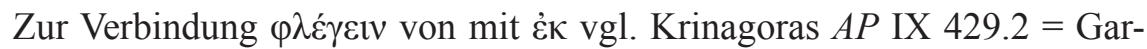

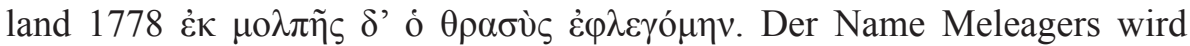
ebenso abgeleitet aus dem Objekt von dessen «Liebesglut» wie der Philodems ( $A P$ V 115.5 s. = Garland 3200 s.):

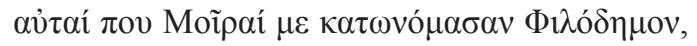

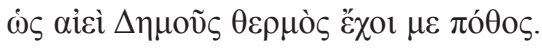


$A P$ XII $84=H E 4602-4609$

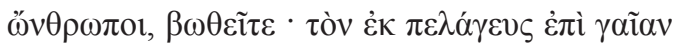

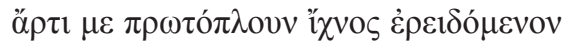

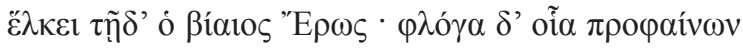

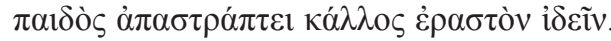

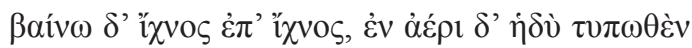

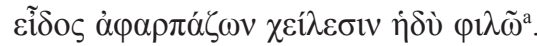

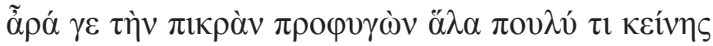

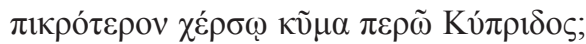

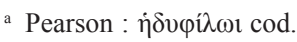

$\mathrm{Zu}$ der Wiederholung von $\dot{\eta} \delta$ bemerken Gow-Page: «The use of a formula ... has misled the poet into inelegance here; the repetition of $\dot{\eta} \delta \dot{v}$ is uncharacteristic and unworthy of him». Auch wenn die Junktur $\dot{\eta} \delta \dot{v} \varphi 1 \lambda \varepsilon \varepsilon \bar{v}$ durch Theoc. 20.1 gedeckt ist, so kann doch an der hier besprochenen Stelle (wo es nur um ein von Eros vorgespiegeltes Luftphantom geht) von einem «angenehm Küssen» wohl kaum die Rede sein: Der Anblick des Phantombilds wirkt auf den Liebenden angenehm, aber bei dem Versuch es zu küssen, wird er seine Nichtigkeit erfahren.

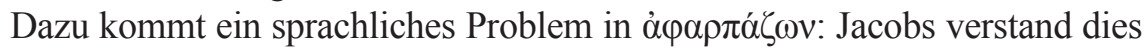
zunächst von eilig gegebenen Küssen («si genuina est lectio, de osculo raptim dato et accepto intellegendum»), bevorzugte dann aber für eine Abänderung in $\dot{\varepsilon} \varphi \varepsilon \rho \pi u ́ \zeta \omega v$. Die Schwierigkeit in der zunächst von Jacobs erwogenen Deutung liegt vor allem darin, daß die «geraubten» Küsse durch keinerlei substantivische Wendung bezeichnet werden. Diese Schwierigkeit war es wohl, die GowPage dazu bewog, Jacobs' Deutung von ả $\varphi \alpha \rho \pi \alpha ́ \zeta \omega v$ zu verwerfen und das Wort stattdessen ohne jede Parallele «of sudden eager clasping» zu verstehen.

Man wird diese Schwierigkeit und den Influenzfehler, der sich in der Wiederholung von $\dot{\delta} \delta$ (was an der zweiten Stelle, wie gezeigt, unpassend ist) bekundet, mit einem einzigen Eingriff zu beseitigen suchen. Alle Schwierigkeiten wären behoben etwa mit ursprünglichem

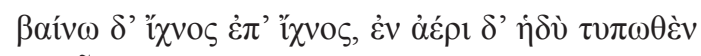

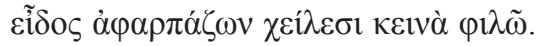




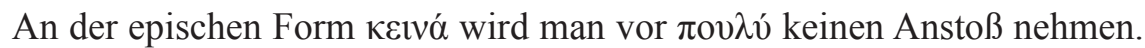

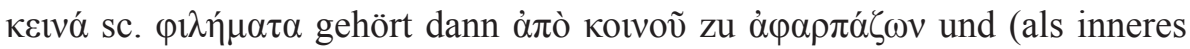
Objekt) zu $\varphi \imath \lambda \tilde{\omega}$.

Bei Meleager vgl. $A P$ XII $125=H E 4628-4635$

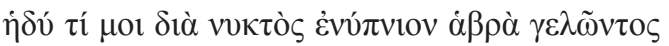

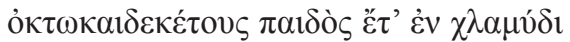

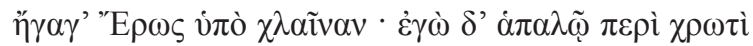

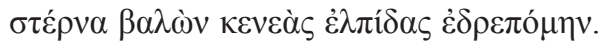

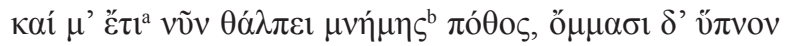

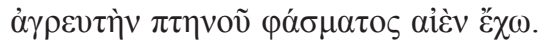

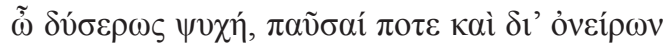

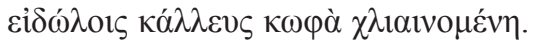

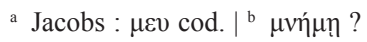

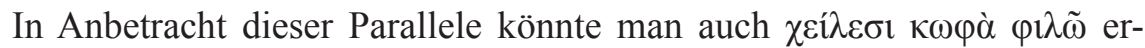
wägen.

Zu $\varphi \imath \lambda \eta ́ \mu \alpha \tau \alpha \kappa \varepsilon v \alpha$ vgl. Theoc. 3.20

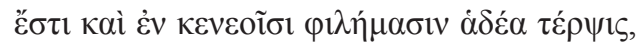

ferner Planudes' Übersetzung von Ou., Met. III 427 Irrita fallaci quotiens dedit oscula fonti! sc. Narcissus:

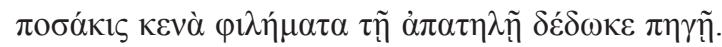

$$
A P \text { XII } 92=H E 4620-4627
$$

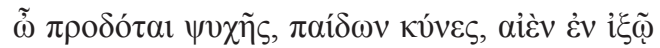

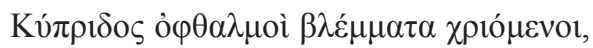

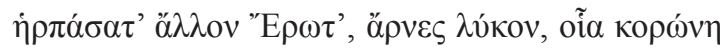
бкорлі́ov, $\dot{\omega} \varsigma \tau \dot{\varepsilon} \varphi \rho \eta \pi \tilde{\nu} \rho \dot{v} \pi \circ \theta \alpha \lambda \pi$ ó $\mu \varepsilon v o v$.

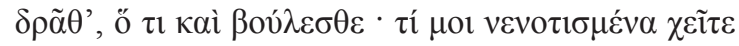

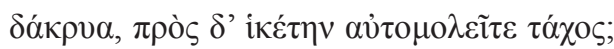

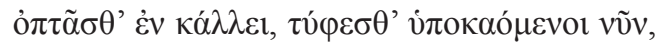

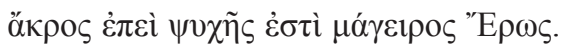




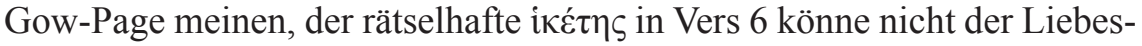
gott sein: «Eros was a wolf and scorpion a moment ago, and is about to roast the soul on a spit; he is anything but a «suppliant»»». Aber diese Ambivalenz macht gerade sein Wesen aus, vgl. AP V $178=H E 4200$ - 4209, wo die bösen und heimtückischen Eigenschaften des Knaben beschrieben werden, die an sich Grund genug sein müßten, ihn als Sklaven zu verkaufen, aber am Schluß sein flehentliches Weinen den Sprecher dazu bringt, von diesem Plan Abstand

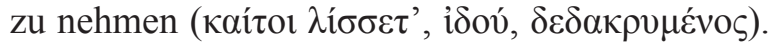

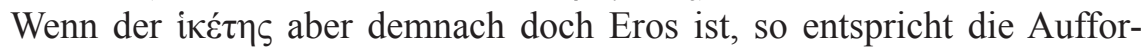
derung der sprechenden Seele an die Augen, das zu tun, was sie eigentlich

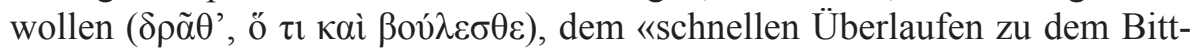
flehenden (sc. Eros)». Man wird $\alpha$ vo $\tau$ )

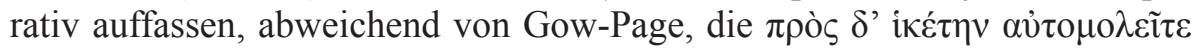

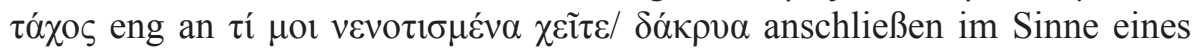
argumentum e contrario (vgl. schon Graefes Paraphrase «quid ad me lacry-

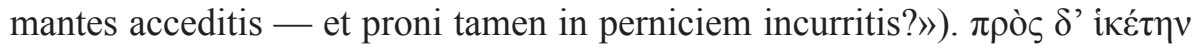

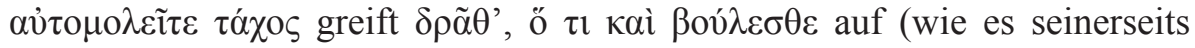
von den höhnischen Imperativen im Schlußdistichon fortgeführt wird), und

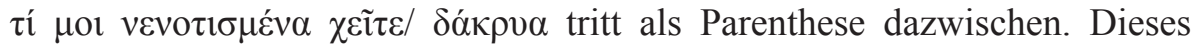

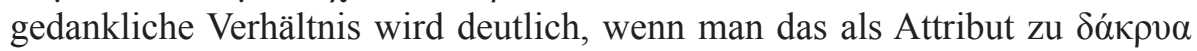

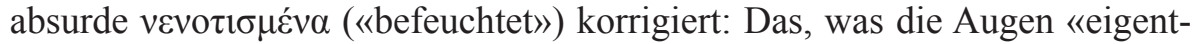

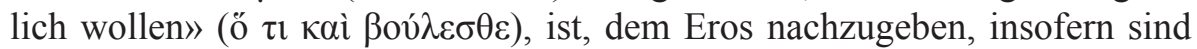
ihre Tränen nur «erheuchelt». Die Parenthese knüpft also unmittelbar an die

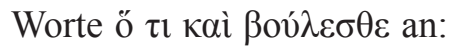

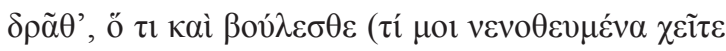

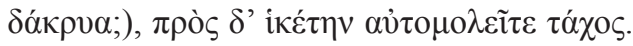

$A P$ XII $157=H E 4642-4645$

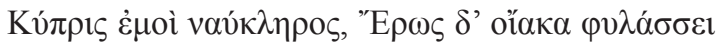

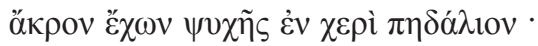

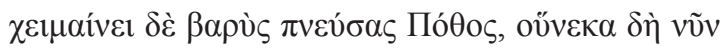

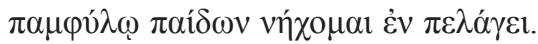

Gow-Page geben $\pi \alpha \mu \varphi v ́ \lambda \omega$ ( $\pi \alpha \mu \varphi \dot{\lambda} \Lambda \omega$ Klotz) die Bedeutungen «of every race and Pamphylian» und nehmen dabei ein «geographical pun» an, welches wohl darauf hinausliefe, daß der Dichter einen pamphylischen Knaben liebte. 
Besser paßt dagegen zum «Meer der Knaben» ein Beiwort, welches einerseits bereits nach homerischem Sprachgebrauch zum Meer gehört (vgl. LSJ s.v. I 1) und andererseits auch in erotischer Sprache die typisch rötliche Hautfarbe eines geliebten Knaben bezeichnen kann (vgl. LSJ s.v. II 2 «of human

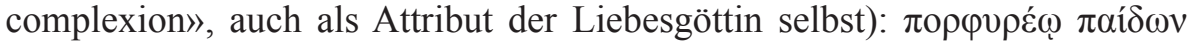

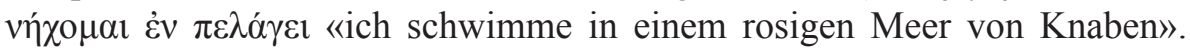

$$
A P \text { VI } 163=H E 4646-4653
$$

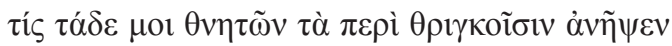

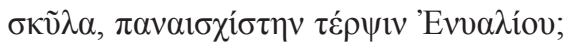

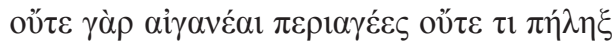

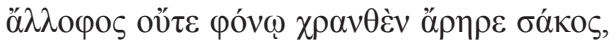

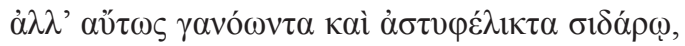

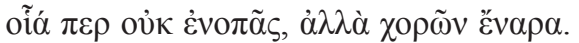

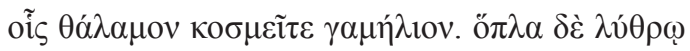

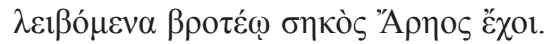

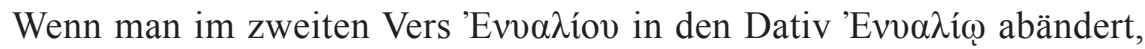
so ergeben sich zwei Vorteile: 1. Durch die syntaktische Kongruenz $\mu$ or ... / ... 'Evva $\lambda i$ ị begreift der Leser viel leichter, daß der Kriegsgott der Sprecher des Epigramms ist. 2. Der für das Gedicht wesentliche Aspekt, daß eine sol-

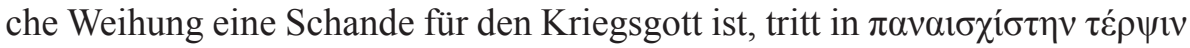
'Evva $\lambda i$ ị deutlich hervor, wohingegen sich der Genitiv 'Evva $\lambda$ íov in sinnwidriger Weise nur mit $\tau \varepsilon \dot{\rho} \psi \mathbf{v} v$ verbindet. Vgl. das Parallelepigramm AP IX 323.3

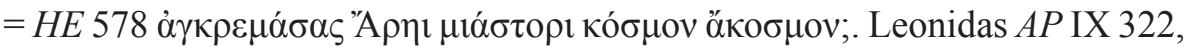

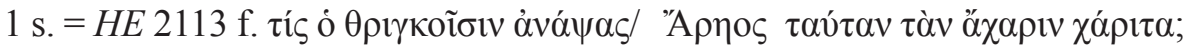

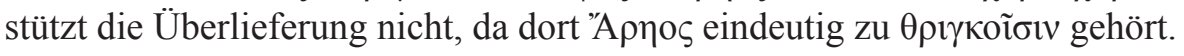

$$
A P \text { VII } 79=H E 4654-4659
$$

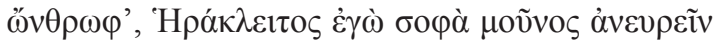

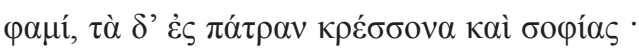

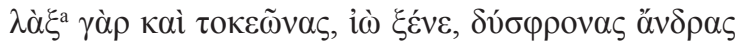

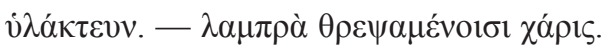

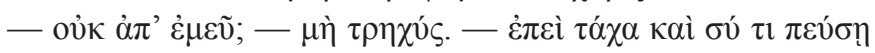

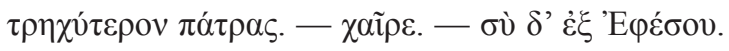

a $\delta \grave{\alpha} \xi$ Jacobs. 
Da sich der Heraklit dieses Grabepigramms seine Verdienste um seine Heimstadt ( $\tau \grave{\alpha} \delta$ ' $\dot{\varepsilon} \zeta \pi \alpha ́ \tau \rho \alpha v$ ) höher anrechnet als seine wissenschaftlichen Ergebnisse, wird er einem frechen Besucher seines Grabs kaum erwidern: «du wirst jetzt gleich Unangenehmeres als meine Heimatstadt von mir zu hören

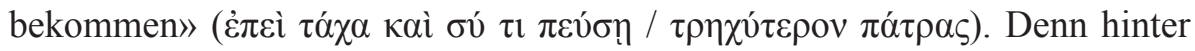
diesem «Unangenehmen» müßte man, wenn der Anspruch in Vers 2 zutrifft, eine «heilsame Lehre» für die Vaterstadt vermuten, die jedoch dem frechen Besucher des Grabs nicht gelten könnte.

Den Hinweis in Richtung einer Emendation gibt die von Headlam aus

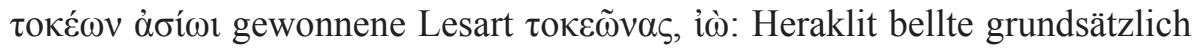

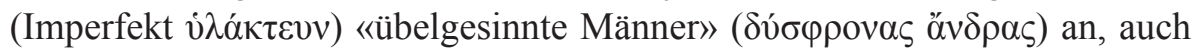
wenn es sich dabei um seine Eltern handelt; zum Motiv vgl. Leonidas $A P$

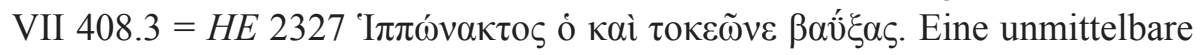

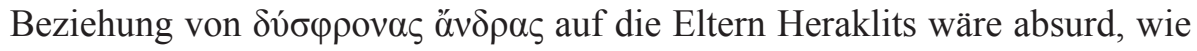
Gow-Page z.St. zeigen.

Vorgeschlagene Lösungen überzeugen nicht: West $C R$ 81, 1967, S. 127 f. nimmt eine - freilich sehr obskure - polemische Wendung Heraklits im Sinne von Fr. 20 D.-K. nicht speziell gegen seine eigenen Eltern, sondern gegen Erzeuger im allgemeinen an (gegenüber Wests neuer Sprecherverteilung im Rest des Gedichts wird hier an Gow-Page festgehalten); Lloyd-Jones $C R$

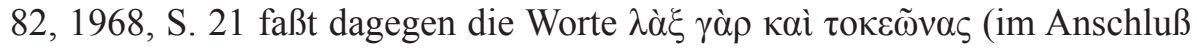
an Wests Sprecherverteilung) als eine Fortführung der Rede des Fremden, die jedoch mit einem syntaktisch unvollständigen Satz ende (im Sinne einer Unterbrechung durch Heraklit oder einer Aposiopese).

Insofern jedoch auch ì̀ $\xi \varepsilon \dot{\varepsilon} v \varepsilon$ statt des üblichen einfachen $\xi \dot{\varepsilon} v \varepsilon$ bedenklich ist (vgl. Gow-Page), empfiehlt sich wohl folgende Herstellung:

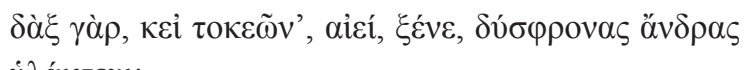
ن̀ $\lambda \alpha ́ \kappa \tau \varepsilon v v . .$.

Denn mit beißender Schärfe pflegte ich stets, Fremder, sogar wenn es meine Eltern waren, übelgesonnene Menschen anzubellen.

Der Dual entspräche dann exakt dem Vorbild Leonidas.

Damit würde auch sehr deutlich, inwiefern das «Anbellen der eigenen Eltern» dem Wohl des Staates dient: Es ist Ausfluß von Heraklits rigoristischer

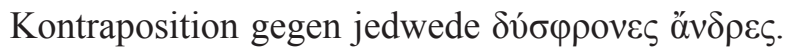


Auf dieses Bekenntnis der Grobheit gegenüber den eigenen Eltern (die freilich einem «höheren Zweck» diente) kontert der Besucher mit einem iro-

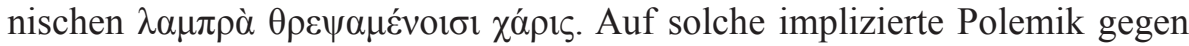
sein Verhalten reagiert dieser wiederum - ganz Fiesling -, indem er dem Besucher angekündigt, er werde bald noch Schlimmeres von ihm als zu hören

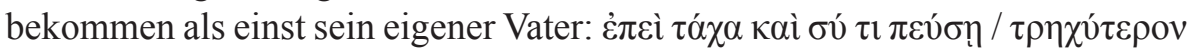

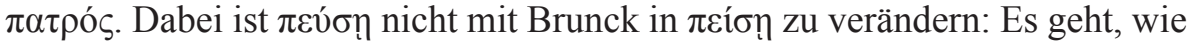

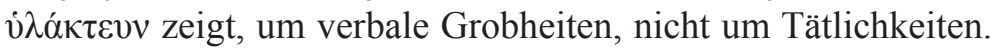

$$
A P \text { VII } 182=H E 4680-4687
$$

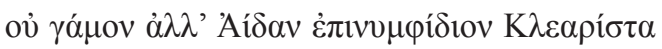

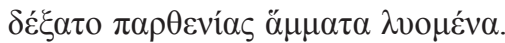

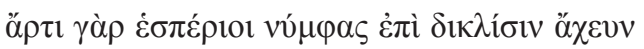

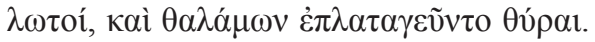

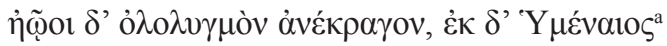

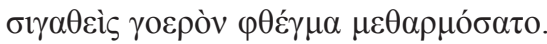

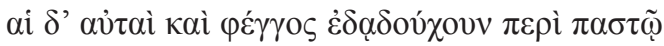

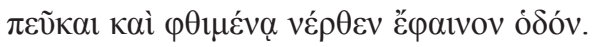

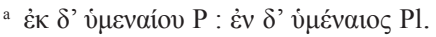

Hymenaios als Subjekt ist gesichert durch das Vorbild Erinna AP VII 712, $7 \mathrm{~s} .=H E 1795 \mathrm{~s}$.

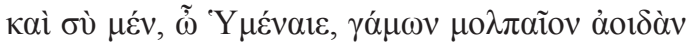

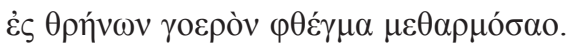

Die bei Stadtmüller referierten zahlreichen Versuche zu $5 \mathrm{f}$. suchen (a) das sonderbare, durch Tmesis getrennte Praefix des sonst nirgends belegten

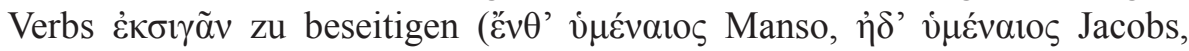

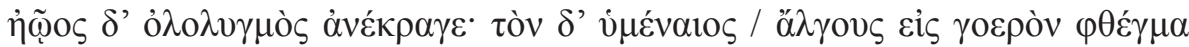
$\mu \varepsilon \theta \alpha \rho \mu o ́ \sigma \alpha \tau$ o Schneider) und/ oder (b) eine exakte syntaktische Analogie zu dem Erinna-Epigramm herzustellen durch Isolation der drei Schluß-

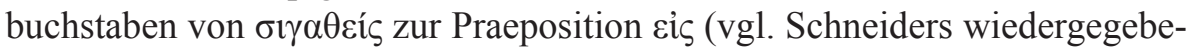

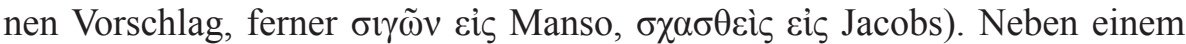
$\mu \varepsilon \theta \alpha \rho \mu o ́ \zeta \varepsilon \sigma \theta \alpha$ $\varepsilon i \zeta$ erwartet man jedoch auch ein Objekt (eine Auffassung 
von $\mu \varepsilon \theta \alpha \rho \mu$ ó $\alpha \tau$ o als direktes Medium in dem Sinne «Hymenaios veränderte sich» empfiehlt sich in Anbetracht des Vorbilds wenig), was man, wenn man nicht zu Schneiders aufwändiger Lösung greifen will, aus $\sigma \downarrow \gamma \alpha \theta$ gewinnen müßte. Schriebe man nun in Analogie zum Vorbild (und in palaeographisch

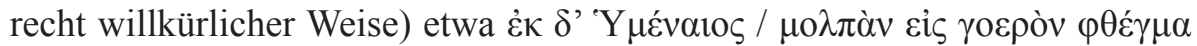

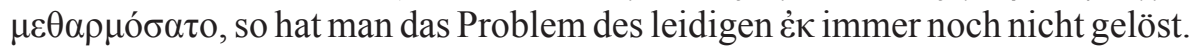

Demgegenüber erscheint die Annahme einer «verdichtenden» Imitationsweise durch $\mu \varepsilon \theta \alpha \rho \mu o ́ \zeta \varepsilon \sigma \theta \alpha \iota$ mit effiziertem Objekt empfehlenswert. Allerdings kann von einem «Schweigen» des Hymenaios dann allenfalls in negierter Form die Rede sein:

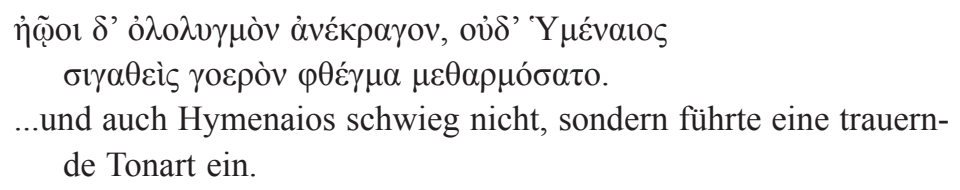

In den beiden Schlußdistichon wird beschrieben, wie die Instanzen einer Hochzeit nicht verschwinden, sondern ihre Funktionsweise ändern: Der

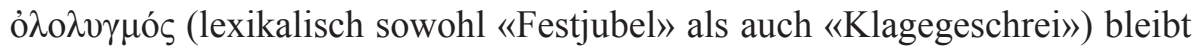
erhalten, auch Hymenaios schweigt nicht, sondern ändert nur seine Tonart, und gleichfalls ändern die Fackeln nur ihre Funktion. oủoź bezieht sich nur

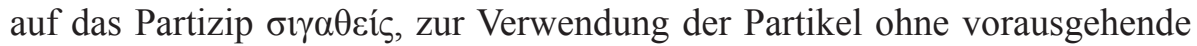
Negation vgl. Denniston 192 («ov̉ó́ for kaì ov̉, simply adding a negative idea to a positive one»).

$$
A P \text { VII } 468=H E 4690-4699
$$

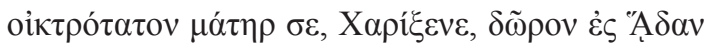

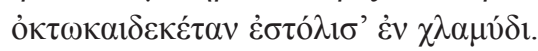

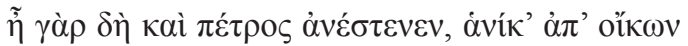

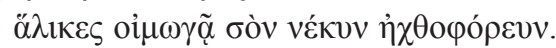

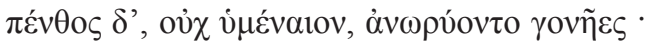

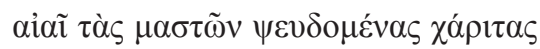

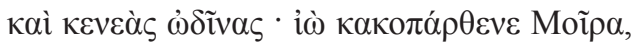

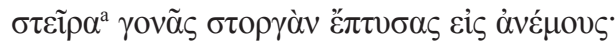

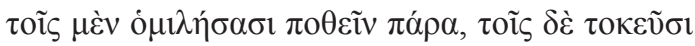
$\pi \varepsilon v \theta \varepsilon \tilde{v} v$, oís $\delta$ '

a Graefe : $\sigma \pi \varepsilon i ̃ \rho \alpha \operatorname{cod}$. 
Gow-Page ziehen $\gamma$ ovã $\varsigma$ zu $\sigma \tau \varepsilon i ̃ \rho \alpha$ und übersetzen «barren of offspring,

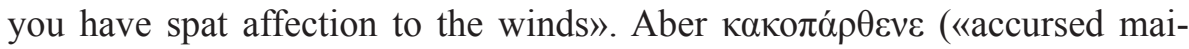
den», Gow-Page) wird erst wirklich verständlich, wenn man $\sigma \tau \varepsilon i ̃ \rho \alpha$ zum

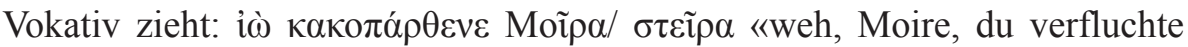
unfruchtbare Jungfrau». Die Moire selbst ist in ihrer virginalen Sterilität nie

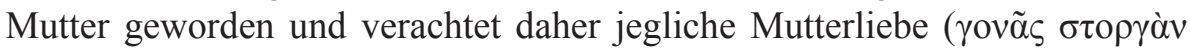

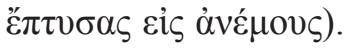

$$
A P \text { VII } 535=H E 4700-4705
$$

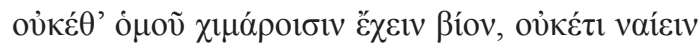

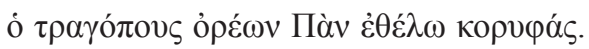

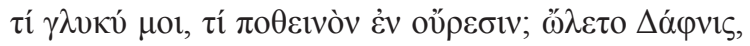

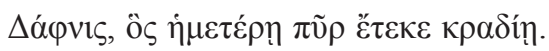

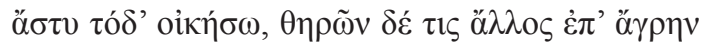

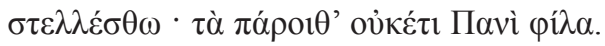

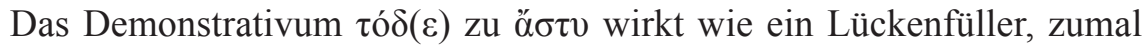
man sich nicht recht vorstellen kann, wo das Epigramm angebracht sein sollte, um diese Deixis zu erklären. Jacobs sagt: «occasionem huic carmini fortasse dedit Panis simulacrum in urbe positum; unde poeta causam fingit, cur deus, rure relicto, in urbem commigraverit», ähnlich Gow-Page «he explains why Pan is content, since Daphnis's death, to live in a town». Aber dann müßte Pan seine Umsiedlung in die Stadt als etwas Abgeschlossenes aitiologisch begründen, nicht aber als eine zukünftige Absicht hinstellen (oỉ bedeuten mir die Berge nichts mehr, daher werde ich hierhin umziehen», wirkt grotesk.

Eine idiomatische gute Ergänzung der wohl unumgänglichen Lükke, die zudem die Wesensentfremdung Pans herausbrächte, wäre ein ő́

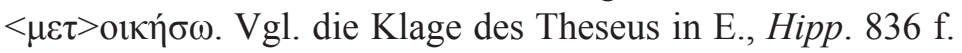

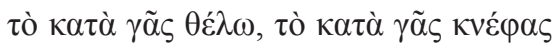

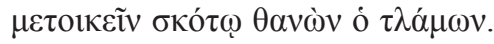

Ein dorisches $\alpha ́ \sigma \tau v<\pi \varepsilon \delta>$ oıkท́ $\sigma \omega$ könnte die Überlieferung gut erklären, liegt aber jenseits der dorischen Dialektpraxis des Meleager. 


$$
\begin{aligned}
& A P \text { IX } 331=H E 4706-4709
\end{aligned}
$$

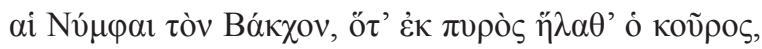

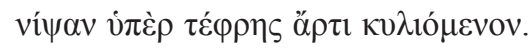

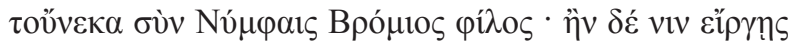

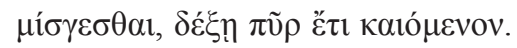

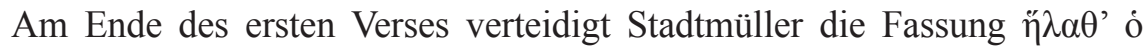

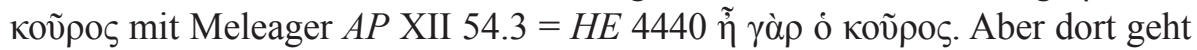
es um einen kontextuell eindeutig bestimmten jungen Mann, hier dagegen um die «Blitzgeburt» des Dionysos, aus welcher dieser offenbar sogleich als Jüngling hervorgegangen ist. Diese praedikative Verwendung zwingt dazu, mit

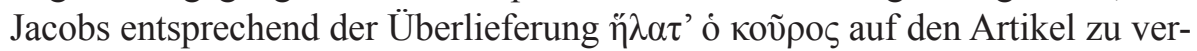

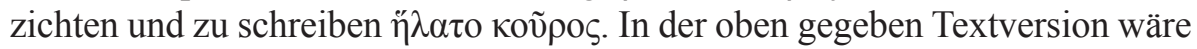

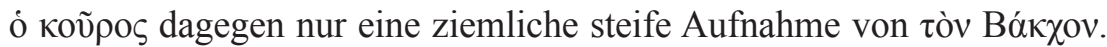

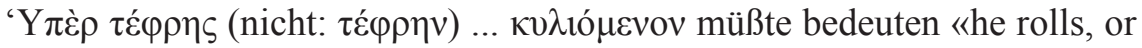
tosses, on top of the ashes» (Gow-Page). Wie man aber jemanden waschen kann, «der sich gerade über der Asche wälzt», bleibt rätselhaft, zumindest wenn ő $\rho \tau \imath$ man scharf von einem zeitlichen Zusammentreffen verstehen will.

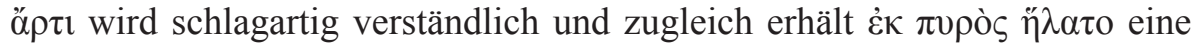
anschauliche Ausgestaltung, wenn man vं $\varepsilon^{\rho} \rho$ in $\dot{\tau} \pi \varepsilon ́ \kappa$ verbessert: «Dionysos

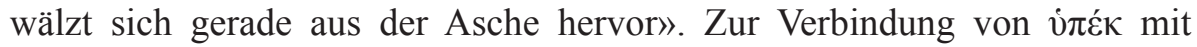

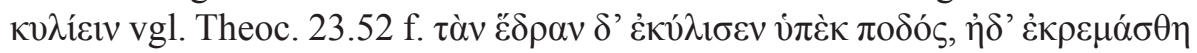

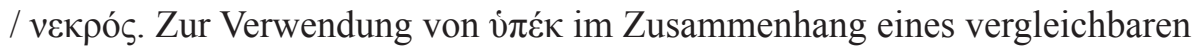

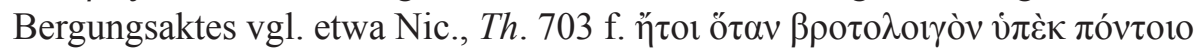

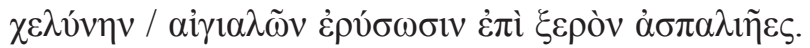

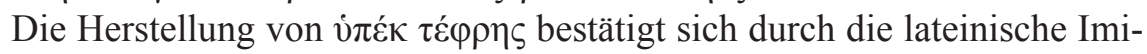
tation $A L 891$ R.:

Infantem Nymphae Bacchum, quo tempore ab igne

Prodiit, inuentum sub cinere abluerant.

Ex illo Nymphis cum Baccho gratia multa est,

Seiunctus quod sit ignis et urat adhuc

(wenn man den letzten Vers ohne konsekutives quod und Kürze in der Dihaerese gestalten will, läge etwa ein Seiunctusque ab eis ignis adurit adhuc nahe). 
In Vers 3 verteidigen Gow-Page die Überlieferung: «бúv is not to be altered ... : The point is not that Bacchus is dear to the nymphs, but that the association of Bacchus with the Nymphs (wine with water) is dear to men». Hiernach erwartet man «wenn man sie (nicht: ihn) an der Mischung hindert», müßte also wohl die recht seltene pluralische Verwendung von viv annehmen.

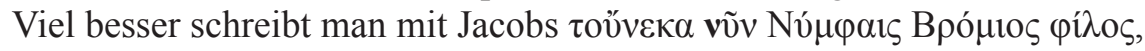
vgl. Ex illo Nymphis cum Baccho gratia multa est. Erst die Temporalpartikel bringt die aitiologisch begründete Konsequenz für die Gegenwart nachdrücklich hervor, und die unmittelbare Folgerung, die man aus der mythischen Erzählung im ersten Distichon ziehen kann, ist eben nicht eine Auffassung der Menschen, sondern die Zuneigung des Dionysos zu den Nymphen. Vor allem aber geht der sexuelle Hintersinn in elpyns/ $\mu$ í $\sigma \varepsilon \sigma \theta \alpha ı$ («am Beischlaf hindern») verloren, wenn nicht vorher von der Freundschaft des Dionysos

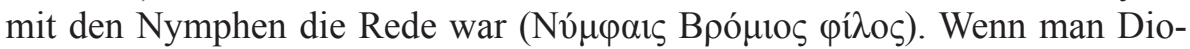
nysos an dieser «Verbindung» hindert, empfängt man ihn «als noch loderndes Feuer». Das ganze Schlußdistichon ist auf Dionysos zugeschnitten, und vvv ist Singular.

Anthologia Planudea $134=H E 4710-4721$

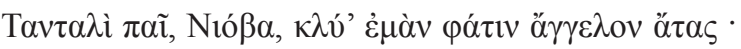

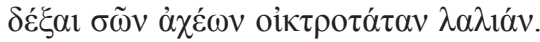

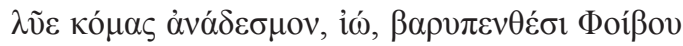

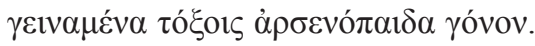

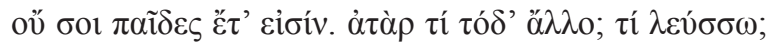

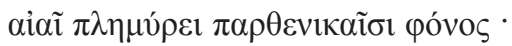

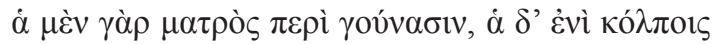

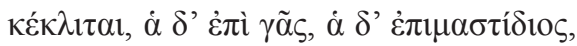

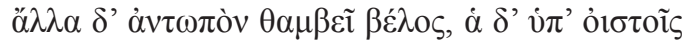

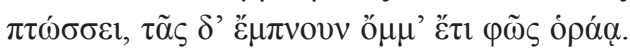

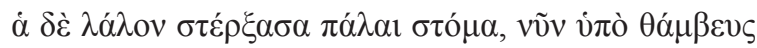

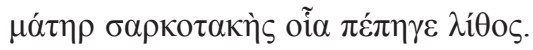

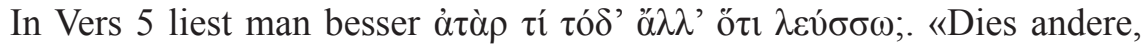
was ich sehe,» tritt in einen gebührenden Gegensatz zu dem vorher erwähnten Unglück, das vom Sprecher hier nur in der Rede erwähnt wird (vgl. દ̇uòv

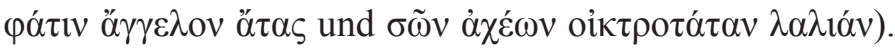


$A P$ VII $352=H E 4742-4749$

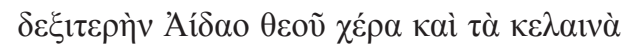

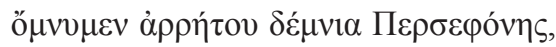

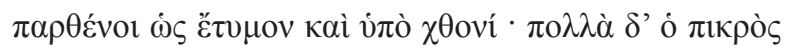

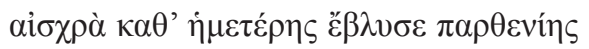

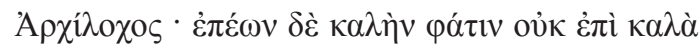

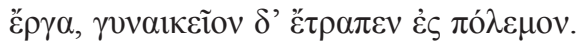

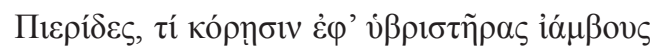

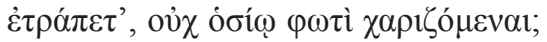

Es kommt nicht darauf an, daß die Lykambiden «Jungfrauen im echten

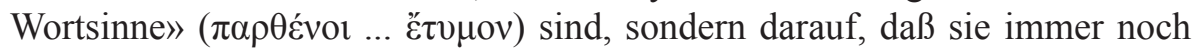

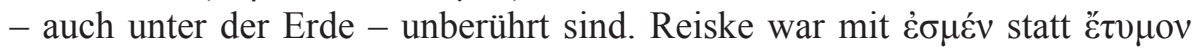
auf der richtigen Spur. Noch praegnanteren Sinn und größere Nähe zur Über-

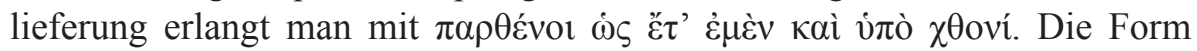
$\dot{\varepsilon} \mu \varepsilon^{\prime} v$ statt $\dot{\varepsilon} \sigma \mu \varepsilon^{\prime} v$ ist abgesichert durch das von einschlägigen Grammatikern

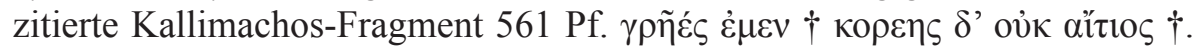

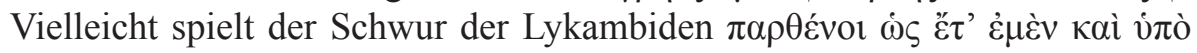
$\chi \theta$ oví irgendwie auf dieses $\gamma \rho \tilde{\eta} \varepsilon \dot{\varepsilon} \zeta \dot{\varepsilon} \mu \varepsilon v$ an. Handelte es sich bei Kallimachos vielleicht um eine ähnliche Tugendversicherung alter Frauen, etwa $\gamma \rho \tilde{\eta} \varepsilon ́ \varsigma$

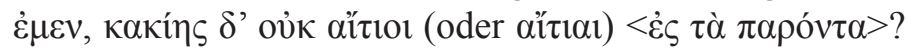

Fecha de recepción de la primera versión del artículo: 26/03/2007

Fecha de aceptación del artículo: 26/03/2007

Fecha de recepción de la versión definitiva del artículo: 06/06/2008 